\title{
Longitudinal optical phonons in the excited state of CuBr quantum dots
}

\author{
Jialong Zhao, ${ }^{*}$ Atsushi Kanno, Michio Ikezawa, and Yasuaki Masumoto \\ Institute of Physics, University of Tsukuba, Tsukuba 305-8571, Japan \\ (Received 12 May 2003; published 24 September 2003)
}

\begin{abstract}
The size dependence of the longitudinal optical (LO) phonons in the excited state of $\mathrm{CuBr}$ quantum dots (QD's) in glass and $\mathrm{NaBr}$ crystals in the intermediate confinement regime was studied by means of persistent spectral hole burning spectroscopy. The phonon-exciton coupled states were clearly observed at a photon energy of about $2.993 \mathrm{eV}$ when the LO phonon energy is close to the energy difference between the ground $1 \mathrm{~S}$ and excited $1 P$ states of CuBr QD's in glass. The energies of the LO phonons observed in smaller CuBr QD's in glass and $\mathrm{NaBr}$ crystals were determined to be about 18.6 and $17.6 \mathrm{meV}$, respectively, which are smaller than that of $\mathrm{LO}$ phonons in the bulk $\mathrm{CuBr}$ material. The energy softening of the LO phonons was explained in terms of the phonon renormalization.
\end{abstract}

DOI: 10.1103/PhysRevB.68.113305

PACS number(s): 68.65. $-\mathrm{k}, 63.22 .+\mathrm{m}, 61.46 .+\mathrm{w}, 71.35 .-\mathrm{y}$

The elementary excitations of small quantum dots (QD's) containing a few hundreds of atoms are expected to modify the lattice vibrational frequencies due to the carrier confinement and resulting strong exciton-phonon interaction similar to the lattice vibrations in the excited state of many molecules and localized centers in solids. ${ }^{1}$ The modification of the vibrational frequency in the electronic excited state of small-sized QD's is so unique that the QD's show a characteristic of the molecular nature. Recently, the energy softening of the longitudinal optical (LO) phonon by $8 \%$ was observed in persistent spectral hole burning (PSHB) and resonant photoluminescence spectra of 2.5 -nm-radius $\mathrm{CuCl}$ QD's, typical QD's in the weak confinement regime. ${ }^{2-4}$ In contrast to the 25.6-meV LO phonon in the ground state observed in Raman scattering and resonant photoluminescence spectra, a $23.5-\mathrm{meV}$ LO phonon was observed in the excited state of $\mathrm{CuCl}$ QD's in glass by means of PSHB and resonant photoluminescence. The exciton-phonon coupled states were clearly observed as a result of strong Fröhlich interaction both in quantum spheres and quantum cubes when the energy of the LO phonon is close to the energy spacings between the ground and excited exciton quantum states. ${ }^{2,4}$ The LO phonon softening in the excited state of $\mathrm{CuCl}$ QD's was also observed by means of quantum beats in the time domain ${ }^{5}$ and two-photon-excited resonant luminescence in the spectral domain. ${ }^{6}$ Theoretically, the energy softening was described in terms of the phonon renormalization in the presence of a single exciton in spherical QD's., ${ }^{2,6,7}$ The theory predicted further the LO phonon renormalization in the excited QD's not only in the weak confinement regime but also in the strong confinement regime. ${ }^{6,8}$

Besides $\mathrm{CuCl}$ QD's, ${ }^{9-13} \mathrm{CuBr}$ QD's are another typical class of semiconductor nanomaterials for studying the quantum confinement effects of the exciton translational motion ${ }^{14-17}$ and cataloged to the intermediate confinement regime on the basis of the ratio of the dot radius to the Bohr radius. $\mathrm{LO}$ phonon modes in the $\mathrm{CuBr}$ bulk material and QD's have been extensively studied by Raman scattering and site-selective luminescence spectra. ${ }^{18-23} \mathrm{Up}$ to now, the sizedependent excited states of the $Z_{1,2}$ excitons in CuBr QD's have not been studied yet. Fortunately, the PSHB phenomenon has been observed in $\mathrm{CuBr}$ nanocrystals, ${ }^{24-26}$ and therefore it provides a sensitive site-selective tool for studying the size-dependent exciton states and phonon modes as well as the formed exciton-phonon coupled states in $\mathrm{CuBr}$ QD's.

In this Brief Report, we extend the study of phonon softening to QD's in the intermediate confinement regime from QD's in the weak confinement regime. We study in detail the LO phonons and exciton states for CuBr QD's embedded in glass and $\mathrm{NaBr}$ crystals in the weak and intermediate confinement regimes by means of PSHB spectroscopy. We successfully found the mixing of the LO phonon with exciton when the energy of the LO phonon approaches the energy spacings between the ground and excited states for $\mathrm{CuBr}$ QD's in glass and the energy softening of LO phonons experimentally for $\mathrm{CuBr}$ QD's in glass and $\mathrm{NaBr}$ crystals.

Samples used in the experiment were CuBr QD's embedded in glass or $\mathrm{NaBr}$ crystals. The average size of the QD's was estimated by small-angle x-ray scattering (SAXS) and transmission electron microscopy (TEM). ${ }^{27}$ The samples were directly immersed in superfluid helium at $2 \mathrm{~K}$ in an optical cryostat. A narrow-band dye laser pumped by the third harmonics of the output of a $Q$-switched $\mathrm{Nd}^{3+}$ : YAG laser $(355 \mathrm{~nm})$ was used as a pump source. The pulse duration and repetition rate were $5 \mathrm{~ns}$ and $30 \mathrm{~Hz}$, respectively. The spectral linewidth was about $0.014 \mathrm{meV}$. A halogen lamp was used as a probe source. The PSHB spectrum was measured as follows: First, the absorption spectrum was obtained and the sample was exposed to dye laser pulses to burn a persistent spectral hole at excitation energy. Then, the absorption spectrum was measured again after the laser exposure was stopped. The absorption spectral change $-\alpha d$ is defined as the difference between the spectra before and after the laser exposure. The transmitted light of the samples was detected by a liquid-nitrogen-cooled charge-coupled device in conjunction with a $75-\mathrm{cm}$ spectrometer involving a $1800-$ grooves $/ \mathrm{mm}$ grating operated in the first order of diffraction. The spectral resolution of the experiment for PSHB spectroscopy was about $0.3 \mathrm{meV}$.

Quantum size effect on the $Z_{1,2}$ exciton in CuBr QD's was observed in independent works, although the size dependence of the $Z_{1,2}$ exciton in CuBr QD's in glass, alkali halides, and polymers are slightly different from each 

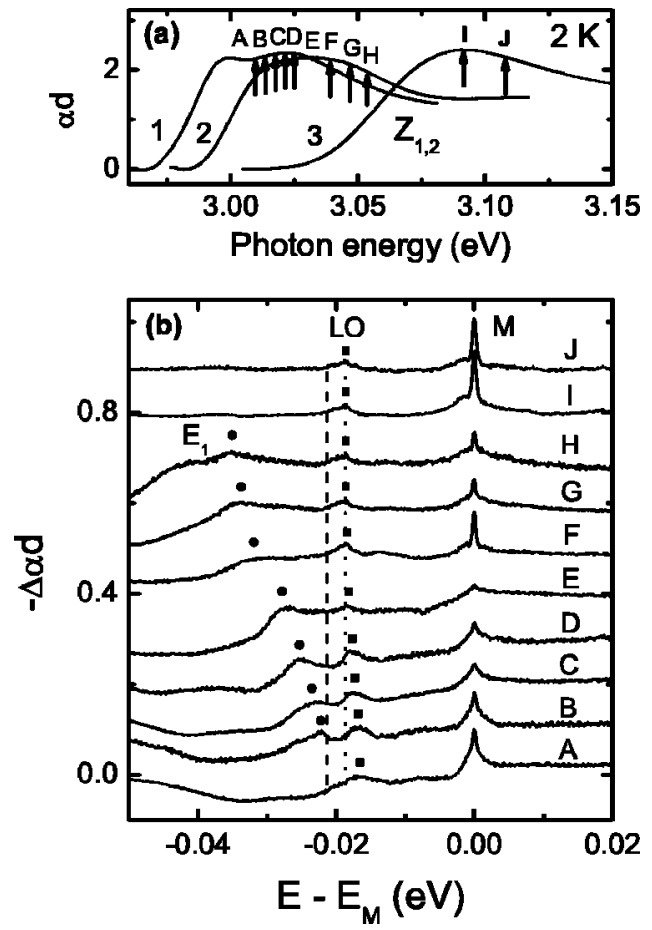

FIG. 1. Absorption (a) and PSHB spectra (b) of CuBr QD's in glass. The average radii for samples 1,2 , and 3 are 10.5, 5.0, and $2.4 \mathrm{~nm}$. The excitation energies for spectra A, B, C, D, E, F, G, H, $\mathrm{I}$, and $\mathrm{J}$ are 3.0094, 3.0130, 3.0167, 3.0203, 3.0242, 3.0388, 3.0463, $3.0537,3.0916$, and $3.1073 \mathrm{eV}$, respectively. The excitation density is $100 \mathrm{~nJ} / \mathrm{cm}^{2}$ and the exposure time is $5 \mathrm{~min}$. The solid and dotted lines in (b) represent the energies of the LO phonons in the ground and excited states of the excitons in smaller CuBr QD's.

other. ${ }^{14-17}$ We investigated the size dependence of the $Z_{1,2}$ exciton in $\mathrm{CuBr} \mathrm{QD}^{\prime} \mathrm{s}^{27}$ and the relation is very close to that reported for CuBr QD's in glass, ${ }^{17}$ which is phenomenologically described by a relation ${ }^{17} \quad E_{n, l}=E_{B}$ $+\hbar^{2} \pi^{2} \xi_{n, l}^{2} /\left(2 M R^{2}\right)+A / R$, where $E_{B}=2.964 \mathrm{eV}$ is the $Z_{1,2}$ exciton energy of a bulk $\mathrm{CuBr}$ crystal at $2 \mathrm{~K}, M=2.6 m_{0}$ is the translational mass of the $Z_{1,2}$ exciton, $R$ and $a_{B}$ are the dot and the Bohr radius, $\pi \xi_{n, l}$ is the $n$th root of the spherical Bessel function of the $l$ th order, ${ }^{10,11}$ and $A$ is a factor. Theoretical treatment of QD's in the intermediate confinement regime shows that weak confinement model holds for the condition $R>4 a_{B}$. On the simple weak confinement model, the size dependence of the energy spacing between the exciton states $1 S$ and $1 P$ in spherical CuBr QD's was simply estimated to be equal to the quantized energy of $1 S$. In the following, we used this model because the anticrossing between LO phonon and $1 S-1 P$ energy split takes place in the large size region, $R>4 a_{B}$.

The absorption and PSHB spectra of CuBr QD's in glass are shown in Figs. 1(a) and (b) at 2 K. As seen in Fig. 1(a), in comparison with the $Z_{1,2}$ exciton energy of the bulk $\mathrm{CuBr}$, by decreasing the size of the QD's, the absorption band of $\mathrm{CuBr}$ QD's in glass is shifted to the high-energy side due to the quantum confinement effect. Further the exciton absorption band of bigger CuBr QD's in glass is found to be split into two peaks probably due to the strain effect. It is clearly noted that the $Z_{1,2}$ exciton absorption band of CuBr QD's is inhomogeneously broadened. This shows that the discrete nature of the exciton states and phonon modes is hidden under the inhomogeneously broadened absorption profile of the CuBr QD's due to the wide distributions in size and shape.

As seen in Fig. 1(b), a sharp main hole marked by $\mathrm{M}$ with asymmetric acoustic-phonon sidebands is burnt resonantly at the excitation energy. The energy of the LO phonon hole for smaller CuBr QD's was estimated to be about $18.6 \mathrm{meV}$. The energies of the LO and transverse-optical (TO) phonons in the bulk $\mathrm{CuBr}$ material are 21.1 and $17.0 \mathrm{meV}$, respectively, which were measured by Raman scattering. ${ }^{18}$ Recently, by resonant Raman scattering, ${ }^{19-22}$ the energy of LO phonon in the $\mathrm{CuBr}$ QD's in glass was measured to be $20.3 \mathrm{meV}$, which was almost the same as that of the bulk $\mathrm{CuBr}$ crystal. The energy of the LO phonon side bands for small 2.4-nm-radius $\mathrm{CuBr}$ QD's in glass was found to be lower than that of the bulk CuBr crystal by $2.5 \mathrm{meV}(12 \%)$. The LO phonon softening observed in CuBr QD's by the PSHB spectroscopy is considered as the LO phonon renormalization in the presence of a single exciton in spherical QD's, in analogy with the LO phonon softening observed in $\mathrm{CuCl}$ QD's. ${ }^{2-6}$ Phonons observed in the PSHB spectroscopy are unique, because it selectively probes the optical phonons in the electronic excited states as a pseudophonon wing at low temperatures. ${ }^{2}$ On the other hand, resonant luminescence observes the optical phonons both in the electronic excited states and in the electronic ground state, and Raman scattering observes the optical phonons in the electronic ground state. The PSHB spectroscopy is superior to others. As seen in Fig. 1(b), a satellite hole marked by $E_{1}$ was observed in the PSHB spectra of larger size dots. The Stokes shift of the satellite hole is almost equal to half of the energy separation between the excitation photon energy and the bulk $Z_{1,2}$ exciton energy. It becomes small gradually when the dot size increases. Thus the satellite hole is considered to come from the hole burning of the ground state $(1 S)$ under excitation of the first excited state $(1 P)$ of the quantum confined excitons in the nearly spherical CuBr QD's.

Figure 2 shows the Stokes shifts of the LO phonon and satellite hole $E_{1}$ as a function of the photon energy of the satellite holes. As seen in Fig. 2, the holes $\mathrm{E}_{1}$ and LO become close to each other when the dot size is increased. When the LO phonon energy of CuBr QD's in glass approaches the energy difference between the $1 S$ and $1 P$ exciton states, the LO phonon energy clearly shows an anticrossing with the Stokes shift of the hole $\mathrm{E}_{1}$ at an energy of about $2.993 \mathrm{eV}$. We consider $1 P$ exciton states can be excited and relaxed to $1 S$ exciton state with the emission of LO phonon $(l=0)$, because CuBr QD's are not perfectly spherical as shown in the TEM image. An anticrossing of the phonon energy with the energy separation between ground and excited state excitons is clearly observed in Fig. 2. The experimental result indicates that the interaction of the LO phonon with the exciton in CuBr QD's in glass results in the formation of the exciton-phonon coupled modes at excitonphonon resonance, which is similar to those observed in $\mathrm{CuCl}$ QD's. ${ }^{2-6}$ On the other hand, as seen in Fig. 2, the 


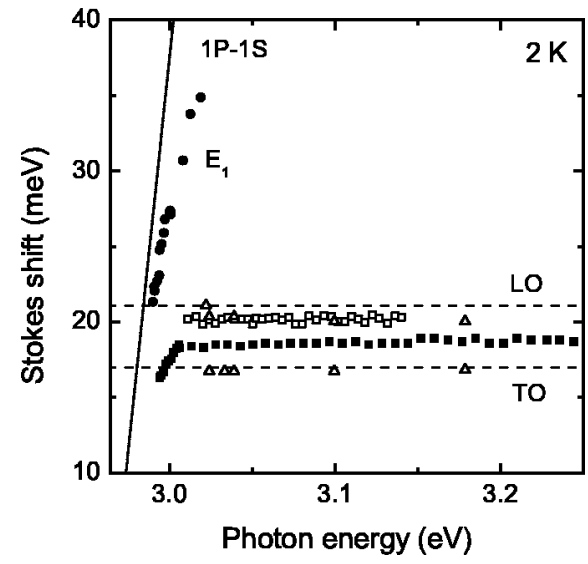

FIG. 2. Stokes shifts of the satellite holes $E_{1}$ (solid circles) and LO (solid squares) in CuBr QD's in glass as a function of photon energy of the satellite holes. The solid line shows the size dependence of the energy difference between exciton states $1 S$ and $1 P$. The dashed lines represent the energies of LO and TO phonons in a bulk $\mathrm{CuBr}$ crystal, respectively. The open squares and triangles show the energies of LO and TO phonons in CuBr QD's in glass, which were measured by Raman scattering (Refs. 19-22).

energies of LO phonons measured by PSHB spectroscopy are clearly smaller than those of LO phonons obtained by Raman scattering. ${ }^{19-22}$

The absorption and PSHB spectra of CuBr QD's in $\mathrm{NaBr}$ crystals are shown in Figs. 3(a) and (b) at $2 \mathrm{~K}$. Unlike $\mathrm{CuCl}$
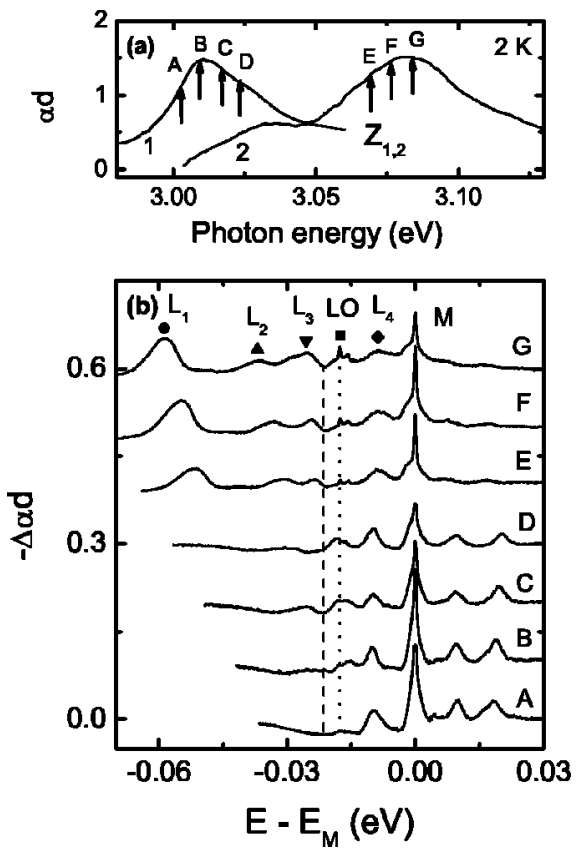

FIG. 3. Absorption (a) and PSHB spectra (b) of CuBr QD's in $\mathrm{NaBr}$ crystals. The average dot radii for samples 1 and 2 are 9.5 and $3.0 \mathrm{~nm}$. The excitation energies for spectra A, B, C, D, E, F, and G are 3.0020, 3.0094, 3.0167, 3.0241, 3.0690, 3.0766, and $3.0843 \mathrm{eV}$, respectively. The excitation density is $100 \mathrm{~nJ} / \mathrm{cm}^{2}$ and the exposure time is $3 \mathrm{~min}$. The solid and dotted lines in (b) represent the energies of the LO phonons in the ground and excited states of the excitons in smaller CuBr QD's.

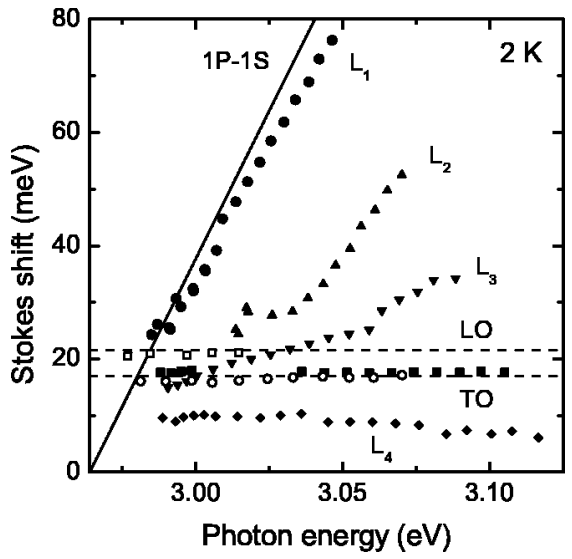

FIG. 4. Stokes shifts of the satellite holes $\mathrm{L}_{1}$ (solid circles) and LO (solid squares) as a function of photon energy of the main hole structures. The solid line represents the energy spacing between the exciton states $1 S$ and $1 P$ in different sized CuBr QD's. The dashed lines show the LO and TO phonon energies in the bulk $\mathrm{CuBr}$ crystal. The open squares and circles show the energies of LO and TO phonons in $\mathrm{CuBr}$ QD's in $\mathrm{NaBr}$ crystals, which were measured by the site-selective luminescence.

QD's in $\mathrm{NaCl}$ crystals, ${ }^{4,13}$ no clear oscillatory fine structure is observed in the absorption spectra of CuBr QD's in $\mathrm{NaBr}$ crystals. As seen in Fig. 3(b), many satellite holes are observed not only at the low-energy side but also at the highenergy side of the main hole M. The Stokes shift of the sharp $\mathrm{LO}$ phonon hole in $\mathrm{CuBr}$ QD's in $\mathrm{NaBr}$ crystals was determined to be about $17.6 \mathrm{meV}$, which is lower than the LO phonon energy in the bulk $\mathrm{CuBr}(21.1 \mathrm{meV})$ by $3.5 \mathrm{meV}$ (17\%) but larger than the TO phonon energy. As seen in Fig. 3 (b), hole $\mathrm{L}_{1}$ is a dominant satellite hole in the PSHB spectra. The Stokes shifts of the satellite holes $\mathrm{L}_{1}$ and LO as a function of the photon energy of the satellite holes are plotted in Fig. 4. The Stokes shift of the hole $\mathrm{L}_{1}$ is considered as the energy difference between the ground $(1 S)$ and excited $(1 P)$ states of the quantum confined exciton in CuBr QD's. If the energy of the LO phonon energy is close to the energy difference, the LO phonon is expected to mix with the excited state of the exciton, which should lead to the formation of the exciton-phonon coupled states. However, we have not observed the anticrossing behavior in large-sized CuBr QD's in $\mathrm{NaBr}$ crystals because we cannot distinguish many overlapped satellite and phonon holes. The exciton-phonon coupled states were clearly demonstrated only in $\mathrm{CuCl}$ QD's in $\mathrm{NaCl}$ crystals ${ }^{4}$ and were not observed in CuBr QD's in $\mathrm{NaBr}$ crystals although the LO phonon softening was observed in both cases. Further, we measured the site-selective luminescence spectra of CuBr QD's in $\mathrm{NaBr}$ crystals at $2 \mathrm{~K}$, in comparison with the energy change of the LO and TO phonons in $\mathrm{CuBr}$ QD's. Some exciton state-related and LO and TO phonon-assisted luminescence bands were clearly observed in the spectra. The energies of LO and TO phonons in $\mathrm{CuBr}$ QD's in $\mathrm{NaBr}$ crystals as shown in Fig. 4 are consistent with those in the bulk $\mathrm{CuBr}$ material. ${ }^{18}$ In addition, it is noted that the many satellite holes from the exciton states were observed in $\mathrm{CuBr}$ QD's in $\mathrm{NaBr}$ crystals but not in glass, which are similar to those in $\mathrm{CuCl}$ QD's in $\mathrm{NaCl}$ crys- 
tals and glass. ${ }^{4,13}$ The reason for this difference possibly results from the QD shape-induced modification of the onephoton selection rules. The Stokes shifts of the satellite holes $\mathrm{L}_{2}$ and $\mathrm{L}_{3}$ may be related to the energy spacings between the higher exciton states in $\mathrm{CuBr}$ QD's with different dot size. However, the phonon-exciton coupled states could not be formed when the LO phonon energy of the ground exciton state $1 S$ is close to these energy spacings.

In summary, we have studied the LO phonons in the excited state of $\mathrm{CuBr}$ QD's in glass and $\mathrm{NaBr}$ crystals. The phonon-exciton coupled states were clearly observed in $\mathrm{CuBr}$ QD's in glass when the LO phonon energy is close to the energy difference between the ground and the first ex- cited states. The energy softening of the LO phonons observed in CuBr QD's was explained in terms of the phonon renormalization with the exciton. These observations of LO phonon softening together with our previous results in $\mathrm{CuCl}$ QD's indicate the universality of LO phonon softening in small-sized QD's.

One of the authors (J.Z.) would like to thank Dr. A. V. Fedorov and Dr. J. Qi for their valuable discussions. SAXS experiments were done by the approval of the Photon Factory (PF) Advisory Committee (Proposal No. 2002G218). This work was partially supported by a Grant-in-Aid for Scientific Research No. 13852003 from the Ministry of Education, Science, Sports and Culture of Japan.
*Present address: Institute of Physical Chemistry, University of Mainz, Welderweg 11, D-55099 Mainz, Germany.

${ }^{1}$ S. Nakajima, Y. Toyozawa, and R. Abe, The Physics of Elementary Excitations (Springer, Berlin, 1980).

${ }^{2}$ L. Zimin, S.V. Nair, and Y. Masumoto, Phys. Rev. Lett. 80, 3105 (1998).

${ }^{3}$ A.V. Baranov, S. Yamauchi, and Y. Masumoto, J. Lumin. 87-89, 500 (2000)

${ }^{4}$ J. Zhao, S.V. Nair, and Y. Masumoto, Phys. Rev. B 63, 033307 (2001).

${ }^{5}$ H. Ohmura and A. Nakamura, Phys. Rev. B 59, 12216 (1999).

${ }^{6}$ A.V. Fedorov, A.V. Baranov, A. Itoh, and Y. Masumoto, Fiz. Tekhn. Poluprovodn. 35, 1452 (2001) [Semiconductors 35, 1390 (2001)].

${ }^{7}$ S.V. Nair and Y. Masumoto, Jpn. J. Appl. Phys., Part 1 38, 581 (1999).

${ }^{8}$ S.V. Nair, L. Zimin, and Y. Masumoto, Proceedings of the 24th International Conference on Physics of Semiconductors, VII-B63, pdf no. 463, Jerusalem, 1998.

${ }^{9}$ A.I. Ekimov and A.A. Onushchenko, Fiz. Tekhn. Poluprovodn. 16, 1215 (1982) [Sov. Phys. Semicond. 16, 775 (1982)].

${ }^{10}$ A.D. Yoffe, Adv. Phys. 42, 173 (1993).

${ }^{11}$ U. Woggon, Optical Properties of Semiconductor Quantum Dots (Springer, Berlin, 1997).

${ }^{12}$ A.I. Ekimov, Al.L. Efros, and A.A. Onushchenko, Solid State Commun. 56, 921 (1985).

${ }^{13}$ N. Sakakura and Y. Masumoto, Phys. Rev. B 56, 4051 (1997).

${ }^{14}$ T. Itoh, Y. Iwabuchi, and T. Kirihara, Phys. Status Solidi B 146, 531 (1988).

${ }^{15}$ A.I. Ekimov, Al.L. Efros, M.G. Ivanov, A.A. Onushchenko, and S.K. Shumilov, Solid State Commun. 69, 565 (1989).
${ }^{16}$ M. Oda, M. Shen, M. Saito, and T. Goto, J. Phys.: Condens. Matter 13, 11465 (2001).

${ }^{17}$ A. Nakamura, M. Ohta, S. Sasaki, and M. Takata, J. Lumin. 7274, 370 (1997).

${ }^{18}$ Physics of II-VI and I-VII Compounds, Semimagnetic Semiconductors, edited by O. Madelung, M. Schulz, and H. Weiss, Landolt-Börnstein, New Series, Group III, Vol. 17, Pt. b (Springer, Berlin, 1982).

${ }^{19}$ A.V. Fedorov, A.V. Baranov, and K. Inoue, Phys. Rev. B 56, 7491 (1997).

${ }^{20}$ K. Inoue, A. Yamanaka, K. Toba, A.V. Baranov, A.A. Onushchenko, and A.V. Fedorov, Phys. Rev. B 54, R8321 (1996).

${ }^{21}$ J. Valenta, J. Moniatte, P. Gilliot, B. Hönerlage, J.B. Grun, R. Levey, and A.I. Ekimov, Phys. Rev. B 57, 1774 (1998).

${ }^{22}$ K. Inoue, A. Yamanaka, N. Tanaka, A.V. Baranov, and A.V. Fedorov, J. Korean Phys. Soc. 32, S569 (1998).

${ }^{23}$ T. Itoh, Y. Iwabuchi, and M. Kataoka, Phys. Status Solidi B $\mathbf{1 4 5}$, 567 (1988).

${ }^{24}$ Y. Masumoto, T. Kawazoe, and T. Yamamoto, Phys. Rev. B 52, 4688 (1995).

${ }^{25}$ For the review see Y. Masumoto, J. Lumin. 70, 386 (1996).

${ }^{26}$ Semiconductor Quantum Dots: Physics, Spectroscopy and Applications, edited by Y. Masumoto and T. Takagahara (Springer, Berlin, 2002).

${ }^{27}$ We carefully investigated the size of CuBr QD's in glass by means of either SAXS or TEM and the energy of the $Z_{1,2}$ exciton absorption peaks at low temperatures. The relation between the average radius of QD's, $R$, and the energy of the $Z_{1,2}$ exciton peaks $E$ is well expressed by a phenomenological equation: $E$ $=B / R^{2}+A$, where $A=2.9989 \mathrm{eV}, B=0.59897 \mathrm{eV} \mathrm{nm}^{2}$, and $R$ is given in a unit of nm. The relation for CuBr QD's in $\mathrm{NaBr}$ was overlapped by that for $\mathrm{CuBr}$ QD's in glass. 